\title{
Abordajes teóricos sobre las transformaciones sociales, económicas y territoriales en las ciudades latinoamericanas contemporáneas
}

Tomás Guevara. Consejo Nacional de Investigaciones Científicas (CONICET), Universidad Nacional de Río Negro (UnRN), Río Negro, Argentina.

RESUMEN | Realiza un recorrido conceptual por diversos abordajes teóricos sobre transformaciones sociales, económicas y territoriales en ciudades latinoamericanas contemporáneas. Para ello, se desarrollan tres de las principales perspectivas presentes en la literatura. El objetivo es analizarlas críticamente e integrarlas posteriormente en un marco conceptual más adecuado. El argumento central es que dichas transformaciones se vinculan con el proceso más general de acumulación capitalista y su reestructuración territorial, por lo que deben ser inscritas en este marco conceptual para evitar la fragmentación teórica. Se propone el materialismo histórico-geográfico como marco analítico que postula que la reestructuración territorial constituye una estrategia del sistema capitalista para reiniciar el ciclo de acumulación en momentos de recesión y crisis. Así, las transformaciones territoriales se inscriben en el universo de mecanismos de ajuste espacio-temporal y se vuelven centrales en la etapa actual del régimen de acumulación capitalista.

PALABRAS CLAVE | reestructuración territorial, globalización, gestión urbana.

ABSTRACT | It reviews a conceptual journey through different theoretical approaches about social, economic and territorial transformations in contemporary Latin American cities. To do this, three of the main views shown in literature are developed. The aim is to analyze them in a critical way and subsequently gather them in a more accurate conceptual framework. The main argument is that such transformations are linked to a more general process of capitalist accumulation and its territorial restructuring, consequently they must be classified within this framework to avoid theoretical fragmentation. Historical geographical materialism is proposed as an analytical framework that states that territorial restructuring makes a strategy from the capitalist system to reset the accumulation process during recession and crisis times. Thus, territorial transformations are part of the variety of mechanisms of spatial and temporal adjustments that becomes in the core of the current stage of the capitalist accumulation regimen.

KEYWORDS | territorial restructuring, globalization, urban management.

Recibido el 26 de agosto de 2013, aprobado el 11 de julio de 2014

E-mail: tguevara@unrn.edu.ar 


\section{Introducción}

Este artículo tiene como objetivo desarrollar diferentes abordajes teóricos sobre los procesos de transformación social, económica y territorial en las ciudades latinoamericanas contemporáneas, para posteriormente criticarlos y proponer su integración en el marco de una perspectiva conceptual que parece más adecuada para la interpretación de dichos procesos.

Lo primero que salta a la vista cuando se recorre la cantidad de trabajos recientes que indagan sobre esta problemática es el innegable auge de los estudios urbanos. Esta profusión es parte de lo que Soja (2008), entre otros, denomina el giro espacial en las ciencias sociales, que está vinculado a lo que el autor seńala como una extendida crisis urbana en los países centrales en la década de 1960. Sin embargo, en cuestión de algunos años, el foco de atención derivó hacia otras temáticas, como los movimientos sociales, las nuevas políticas sociales y económicas propuestas por el Consenso de Washington, la transición de los países de la ex Unión Soviética, etcétera. Hubo que esperar hasta mediados de la década de 1990 para que, nuevamente, algunos de los principales cambios y problemas sociales comenzaran a ser analizados desde una perspectiva específicamente espacial.

Desde entonces, se han multiplicado los trabajos y los abordajes en esta área. Sin embargo, como también señala Soja (2008), este auge de los estudios urbanos estuvo caracterizado por un sesgo casuístico y una cierta reticencia a generar conceptos y abstracciones más generalizables, lo que dificultó la condensación de perspectivas teórico-conceptuales acabadas. Primó entonces la diferenciación conceptual, que llega a extremos que hacen imposible que decante un corpus más o menos acabado de conceptos y teorías que permitan delimitar con mayor claridad una disciplina.

En este contexto, sin pretender generar dicha acabada síntesis conceptual en el campo de los estudios urbanos, pero con el afán de ordenar algunos de los principales abordajes teóricos sobre transformaciones en las ciudades latinoamericanas contemporáneas, se presentan algunas de las principales perspectivas analíticas identificadas.

\section{Globalización, ciudades globales y ciudades duales}

Una de las principales líneas de investigación sobre transformaciones en ciudades latinoamericanas contemporáneas es aquella que las asocia estrechamente al fenómeno de la globalización, entendido como una intensificación de los intercambios económicos a escala global -es decir, no sería un fenómeno novedoso, sino la profundización de una tendencia y de la conciencia de este cambio-, con importantes consecuencias en las relaciones sociales, económicas, políticas y culturales. Este abordaje pone las transformaciones territoriales como variable dependiente de un conjunto de transformaciones sociales y económicas de nivel global, que se traducen espacialmente en la modificación del rol y las funciones de los espacios urbanos.

Según este abordaje, la globalización económica que se inicia en la década de 1970 , liderada por el capital financiero, tiene como una de sus principales consecuencias la reformulación de la división internacional del trabajo, que implica la reestructuración territorial de la producción industrial. Esta se desplaza desde los 
países centrales hacia los nuevos centros industriales en el Sudeste Asiático y, en menor medida, hacia algunos países de Latinoamérica, en búsqueda de mejores condiciones de acumulación. En este contexto, la inversión extranjera directa es uno de los principales vectores a través de los cuales se globalizan ciudades y territorios periféricos.

Desde esta perspectiva, la globalización genera impactos directos en el rol desempeñado por las ciudades, reformulando su papel tradicional como espacios de producción y poniendo de relieve su condición de espacios de control y dirección del nuevo régimen de acumulación, con una preponderancia de los llamados "servicios avanzados de la producción". Emergen así las ciudades globales, que se autonomizan relativamente de su territorio adyacente, poniendo en jaque la noción de Estado nacional como contenedor y unidad de análisis de los procesos sociales ${ }^{1}$. Las ciudades globales se configuran como territorios en los que lo global se inserta directamente, pasando por alto la escala nacional (Sassen, 2007), y que van configurando una red jerárquicamente organizada y con una clara división del trabajo. Esta red de ciudades da origen a una "nueva geografía de la centralidad", que rompe con la dicotomía tradicional sur-norte o centro-periferia.

Castells (1995) analiza el mismo fenómeno desde la óptica del modo de producción y postula entonces el pasaje a la "sociedad red" en el marco de la conformación de la "era de la información". El autor afirma que asistimos a la emergencia de un nuevo modo de desarrollo denominado informacional, que estaría caracterizado por una nueva lógica espacial, a la cual llama el "espacio de los flujos", y por una nueva lógica del "tiempo atemporal". La ciudad global, según este autor, no es tanto un lugar, como un proceso que conecta los segmentos más dinámicos de la economía. La red se constituye en la unidad básica de este tipo de sociedad y es el modelo organizacional paradigmático de los actores económicos, sustituyendo a la gran empresa integrada verticalmente.

La reformulación del rol de las ciudades tiene impactos directos en su estructura territorial. Es por ello que en ese marco emerge también la noción de "ciudades duales" (Castells \& Mollenkompf, 1992), que apunta a dar cuenta de la inserción parcial de los territorios en la dinámica económica global y sus efectos en la estructura social y económica, fundamentalmente a través de la segmentación de los mercados laborales (Borja \& Castells, 1997). Es en estos espacios globalizados donde se observan mejores niveles de ingreso, las mejores condiciones habitacionales y de calidad de vida, etcétera. Pero, de la misma manera, la inserción en la globalización genera una marcada tendencia hacia la polarización social, promoviendo marginalidad y exclusión en los territorios que no logran insertarse plenamente en el nuevo orden mundial. Marginación y exclusión se convierten en la contracara del desarrollo económico y no, como en el esquema desarrollista tradicional, en un estadio inferior del desarrollo de las sociedades. El principal vector de esta polarización es el mercado de trabajo, a través del desempleo, pero también de la precarización laboral en diversas modalidades: subempleo, precarización,

También se ven afectados por el surgimiento de entidades supranacionales que erosionan su autoridad "por arriba". 
flexibilización, tercerización, etcétera. Es por eso que a la nueva geografía de la centralidad se contrapone, como su contracara, una "nueva geografía de la marginalidad", compuesta por aquellos lugares que son excluidos de los beneficios de las dinámicas de la globalización (Sassen, 1997).

Uno de los corolarios de la perspectiva de la globalización es la importancia creciente de la escala local en los análisis urbanos, como contracara de las tendencias globalizadores. Global y local representan dos caras de la misma moneda: el reforzamiento de las tendencias globalizadoras impone una reconfiguración de la escala local. De ahí que se multipliquen los estudios de caso orientados a traducir en escala local los procesos globales. La gestión urbana sufre profundas modificaciones, en el contexto de declive de las nociones keynesianas y el auge de las políticas de libre mercado. Se produce una competencia creciente entre las ciudades por captar las inversiones directas, lo que ha determinado la obsolescencia de la planificación normativa y dado lugar a la subsidiaridad del Estado, que ha desregulado progresivamente la gestión urbana (De Mattos, 2002). Como consecuencia, la definición del uso del suelo fue dejada en manos del mercado en función de las rentabilidades relativas de las localizaciones y las actividades. El suelo urbano cobró mayor importancia como medio de valorización de capitales y el plan urbano cede lugar al plan estratégico, herramienta de gestión urbana paradigmática del "urbanismo globalizador".

Esta perspectiva de abordaje de los procesos de transformación social, económica y territorial presenta algunos problemas, desde nuestra perspectiva. En primer lugar, define una etapa completamente nueva determinada por la intensificación de tendencias preexistentes, lo cual no parece del todo atinado. Antes bien, sería más razonable suponer que lo que se denomina globalización no es sino la reformulación de algo preexistente y que responde a una dinámica estructural del sistema capitalista: su tendencia expansiva, que ya había sido identificada por Marx y Engels desde el Manifiesto Comunista (1848). De hecho, pueden mencionarse numerosos antecedentes históricos de autores que señalaron esta tendencia a la emergencia de una escala global en términos económicos y sociales, donde la ciudad desempeña un papel preponderante: desde las ciudades mundiales de Spengler (1918), pasando por los sistemas urbanos de escala global de Friedmann (1972). No quiere decir que no haya novedades dignas de ser destacadas -como la revolución tecnológica-, pero no se han modificado sustancialmente las características de la mundialización capitalista. De hecho, en trabajos posteriores, Sassen (2010) reconoce que, en términos generales, la etapa en curso no es más que una nueva fase del sistema capitalista, lo que lleva a matizar sus afirmaciones.

En el mismo sentido, Castells (1995) afirma que el modo de producción capitalista sigue vigente mientras que, por el otro lado, también afirma la emergencia de un nuevo tipo de sociedad, como si modo de producción y tipo de sociedad pudieran ser escindidos. En otros pasajes, para salvar esa falsa distinción, define esta transición como la emergencia de un nuevo modo de desarrollo. Según el autor, así como las economías capitalistas pasaron de un modo de desarrollo agrario a uno industrial, ahora estaríamos siendo testigos de una transición desde el modo de desarrollo industrial al informacional. Pero, cuando se pone a detallar este nuevo 
modo de desarrollo, no queda claro qué es lo que lo diferencia del modo anterior, ya que, como reconoce el propio autor, el conocimiento y la información siempre cumplieron un rol central en el proceso productivo.

En este sentido, también es necesario matizar la afirmación que postula la emergencia de una nueva geografía de la centralidad. De hecho, se podría argumentar que las ciudades que ocupan los principales lugares de la red global de ciudades son prácticamente las mismas que lideraron la economía en etapas previas. Lo realmente inédito de esta etapa es la reestructuración productiva, que determina la relocalización industrial en países periféricos, más que la reformulación del liderazgo de ciudades como Londres o Nueva York. Así, la verdadera novedad de esta etapa en términos de geografía de la centralidad es el ascenso del polo del Pacífico Asiático (China, tigres asiáticos), estrechamente ligado a la mencionada reorganización productiva.

Por otra parte, el abordaje de las ciudades globales es bastante apropiado para caracterizar las ciudades que analiza Sassen, pero difícilmente puede ser generalizado más allá de un puñado de ciudades de países centrales. De esta manera, se postula que las ciudades se autonomizan de sus Estados nacionales, desconociendo el rol fundamental de algunos de ellos como promotores del crecimiento económico en países como Brasil, Rusia, India o China. A los fines de este trabajo, es seguro que la categoría de ciudades globales y toda la carga analítica que estas conllevan, no puede ser aplicada con rigurosidad a la realidad de ninguna ciudad latinoamericana contemporánea. A lo sumo, como señala Pradilla Cobos (2009), las más importantes ciudades de la región, como México D.F., San Pablo y Buenos Aires, pueden ser interpretadas como los nodos o las correas de transmisión que permiten el drenaje de recursos desde los países periféricos de la región hacia los países centrales, en un esquema centro-periferia que si bien reformulado y complejizado, todavía está vigente. Incluso esta afirmación podría ser matizada, dado que la extensión de formas precarias de industrialización, como la maquila o la industria de ensamblaje en países como México o Argentina, ni siquiera se localiza de forma exclusiva en dichas grandes ciudades, sino que a veces lo hace en ciudades intermedias que se ven favorecidas por su localización -como Tijuana o Ciudad Juárez en México- o por regímenes de promoción -como Ushuaia en Argentina-.

Existen innegables novedades en todo el proceso descrito por los autores que se inscriben en esta perspectiva, como la reestructuración productiva que dispersa territorialmente y reintegra simultáneamente, a través de redes, la unidad del proceso de producción; la emergencia de una unidad económica global en los mercados financieros, que opera en tiempo real y que dota de una movilidad extrema al capital frente a la inmovilidad relativa del trabajo; la centralidad del conocimiento y la información como factores de productividad y competitividad; la desagregación del trabajo, individualizado y fragmentado por los cambios organizativos de la nueva empresa red; etcétera. No obstante, todos estos cambios pueden ser mejor entendidos como el producto de un nuevo régimen de acumulación del capitalismo industrial, en vez de superponer conceptos y teorías no adecuadas para comprender cabalmente los fenómenos y sus múltiples manifestaciones. 


\section{Segregación social}

Existe una segunda línea de investigación que interesa destacar, la cual, a diferencia de la anterior, se centra más en los aspectos sociológicos de las transformaciones territoriales, haciendo hincapié en la localización de los diferentes sectores sociales en el espacio y la relación entre sus localizaciones. Esta perspectiva es la de la "segregación”, una problemática que tiene una gran tradición en los estudios urbanos. Se pueden rastrear en los trabajos pioneros del siglo xix de Engels (1848) y Booth (Lanzetta, 2011). El primero analiza, desde una perspectiva etnográfica, la lógica de producción del espacio de las ciudades del capitalismo industrial en Inglaterra. En el caso de Booth, la perspectiva etnográfica es reemplazada por la mirada cenital, donde a partir de estadísticas se busca mapear las condiciones habitacionales de la población (Lanzetta, 2011).

Posteriormente, la segregación fue recuperada por la Escuela de Chicago, una de las primeras escuelas de investigación en abordar la ciudad como un objeto de estudio. Conceptualizada como la distribución desigual de los individuos en el espacio, la segregación va a ser problematizada en términos de segregación racial, vinculada a los procesos de migración rural-urbano de la población afroamericana del sur de los Estados Unidos. La segregación, según la Escuela de Chicago, fue entendida como el resultado de las preferencias individuales (Rodríguez Merkel, 2011).

La perspectiva de la segregación racial está estrechamente relacionada con la conceptualización del "gueto" por parte de la Escuela de Chicago. El gueto es analizado como un área natural, donde la población de raza negra se localiza como primera residencia en la ciudad. Esta primera conceptualización no tiene necesariamente la carga negativa que se le asignó con posterioridad e implica un conjunto de instituciones y de redes sociales que hacen a la integración de la población migrante. Concretamente, Wacquant (2007) define el "gueto comunitario" como una serie de mecanismos institucionales de control etno-racial, que acoge a todo el abanico de clase sociales de dicho grupo etno-racial. Es una institución de exclusión racial, pero no necesariamente socioeconómica. En la actualidad, Wacquant (2010) señala la transición del gueto al "hipergueto", que implica una decadencia del gueto comunitario, combinando la exclusión racial con la exclusión socioeconómica vinculada al desempleo masivo, la desertificación institucional, el abandono del Estado de bienestar, etcétera.

En las ciudades latinoamericanas contemporáneas, el estudio de la segregación estuvo orientado fundamentalmente por los clivajes socioeconómicos, en detrimento de los clivajes de raza o etnia (Rodríguez Merkel, 2011). Sabatini (1999) la define como "aglomeración geográfica de familias de una misma condición o categoría social” (citado en Rodríguez Vignoli, 2001, p. 12), sea que esta se defina en términos sociales, raciales u otros.

Existen dos abordajes principales sobre la problemática que, de alguna manera, remiten a los abordajes pioneros de Booth y Engels, ya señalados. El primero se orienta a la medición del fenómeno, operacionalizándola a partir de variables socioeconómicas, provenientes de censos de población y vivienda, fundamentalmente. La forma más habitual de abordar la segregación en este caso es a partir 
de la localización residencial y el nivel socioeconómico de los diferentes grupos sociales, utilizando para ello variables de ingresos, ocupación y/o educación. Abordajes de este tipo se pueden encontrar en los trabajos de Rodríguez Merkel (2011), Groisman y Suárez y (2006) sobre ciudades de Argentina; Sabatini (1999), Sabatini, Cáceres y Cerda (2001) y Sabatini y Brain (2008) sobre Chile; Kaztman (2001) sobre Uruguay, etcétera.

Una cuestión central en este abordaje está referida a la reflexión sobre las escalas de la segregación, que puede ser barrial, local, regional, nacional. La escala de análisis elegida es muy relevante, porque puede generar problemas vinculados a la unidad espacial de medición y precipitar conclusiones que ocultan situaciones de "falsa mixidad" (Rodríguez Merkel, 2011). Ward (2001), por ejemplo, afirma que la segregación a escala macro no se ha intensificado en América Latina, pero sí lo han hecho la fragmentación y la segregación a una escala micro. Indicios similares encontraron para el caso del Gran Buenos Aires Groisman y Suárez (2006). Tendencias opuestas, en cambio, observó Ibarra Cofré (2007) para el caso de Canela (Rio Grande do Sul, Brasil), en lo que sería una contratendencia propia de las ciudades de orientación turística. No existe una conclusión acabada en la literatura sobre cuál es la escala de análisis más apropiada para el abordaje de la segregación, lo que genera bastante confusión en cuanto a los diagnósticos referidos a la problemática.

El segundo abordaje, más cercano a la etnografía, se ocupa del estudio en profundidad de un área delimitada definida como segregada, la cual puede ser un barrio, una villa, un asentamiento. Lo que se busca en este caso es la caracterización de la forma de vida de la población en un área segregada y, en el mejor de los casos, su vinculación con el resto de la sociedad. Este enfoque presenta algunas similitudes con el utilizado en los trabajos de Wacquant sobre "áreas de relegación” (2007) y se puede encontrar en una multiplicidad de estudios sobre urbanizaciones populares en Latinoamérica. Lo interesante de estos abordajes es que la mayoría parte de definir a priori un área como segregada, excluida o socialmente aislada (como en Suárez, 2005). La definición de área segregada opera como un presupuesto fuerte para la investigación, a partir del cual se enfatiza en la sociabilidad, las redes sociales e instituciones del barrio, el acceso al mercado de trabajo y a los servicios y bienes públicos, en un intento de desentrañar los mecanismos que reproducen y/o combaten la segregación y el aislamiento (Ibarra Cofré, 2007).

Si bien existe consenso sobre los efectos negativos de la segregación cualquiera sea la forma en que se la aborde, no existe consenso, en cambio, sobre cómo realizar diagnósticos adecuados sobre ella. En líneas generales, se entiende que un nivel elevado de segregación tiene efectos nocivos en términos de las estructuras de oportunidades de vida de la población segregada (Kaztman, 1999), ya que tiende a perpetuar las condiciones de pobreza, limitar la posibilidad de acceso a redes sociales y a determinado capital social y simbólico que promuevan el ascenso social o la integración. De este entendimiento se derivan políticas públicas que buscan promover una mayor mixidad del tejido urbano en términos socioeconómicos. No obstante, también existen evidencias de las consecuencias negativas -como el aumento de la violencia y la delincuencia- que puede traer la coexistencia en un mismo espacio de sectores socioeconómicos muy alejados en la estructura social, especialmente 
cuando a esta distancia social se le añade una evidente fragmentación de la trama urbana. El ejemplo más típico en las ciudades latinoamericanas contemporáneas puede ser la coexistencia, en tierras periféricas de expansión, de urbanizaciones cerradas con barrios populares de origen informal. En definitiva, si bien el abordaje teórico de la segregación arroja mucha información interesante sobre la localización de los diferentes sectores en el espacio urbano y las formas de interrelación de los mismos en casos de estudio puntuales, tal enfoque no llega a dar cuenta de forma acabada de la lógica de producción y transformación del mismo. En este sentido, no problematiza la dimensión específicamente territorial de las transformaciones en curso, sino que se limita a analizar sus efectos en términos sociales. Esto no conduce a una adecuada integración de la dimensión territorial en el análisis, la cual se reduce a ser incorporada como contexto o escenario de los procesos sociales analizados. Tal es una de las principales limitaciones de la segregación como perspectiva de análisis de las transformaciones de las ciudades latinoamericanas contemporáneas.

\section{Gentrificación y difusión urbana}

En tercer lugar, existen numerosos autores que abordan la problemática de las transformaciones sociales, económicas y territoriales haciendo hincapié en los elementos físicos y morfológicos que adquieren estos cambios, a los que imputan procesos sociales que les darían sustento material. En este caso, el énfasis está puesto en las transformaciones de áreas o sectores de la ciudad o en la lógica espacial que adquiere el crecimiento y la expansión de las mismas. Si bien en este enfoque pueden reconocerse múltiples perspectivas y conceptualizaciones, en un intento de sistematizarlas pueden ser integradas en dos sublíneas de investigación: el enfoque en la gentrificación, y aquel centrado en los procesos de difusión urbana.

\section{Gentrificación}

En primer lugar, existe una sublínea de investigación que da cuenta de las transformaciones que acontecen en las áreas centrales de las ciudades. Esta línea se ocupa de caracterizar y explicar los llamados procesos de "gentrificación", que si bien implican dinámicas sociales y económicas específicas, parten de un énfasis especial en las características físicas del proceso. El término fue originalmente acuñado por Ruth Glass (1964, citado en Slater, 2011) para caracterizar transformaciones muy particulares en el área central de Londres de la década de 1960, pero fue ampliamente aceptado por investigadores de diversas latitudes. En su acepción más clásica, describe la renovación de las áreas centrales degradadas de las ciudades, habitadas por la clase trabajadora, a partir de la localización de sectores de la alta burguesía (gentry). El proceso trae aparejada una revitalización del estilo de vida urbano del centro, en contraposición con el modelo de suburbanización clásico y la aparición de nuevos estilos de vida.

Se pueden reconocer dos grandes vertientes explicativas para los procesos de gentrificación. La primera, cuyo autor más representativo es Ley (1986), desarrolla una explicación del fenómeno de gentrificación haciendo hincapié en el componente de la demanda, es decir, cómo es que surgen los agentes gentrificadores. Su 
explicación se basa fundamentalmente en los cambios en el mercado laboral y la estructura social vinculados a la emergencia de una economía posindustrial. La aparición de una clase vinculada a los servicios de esta nueva economía es la base material que da sustento a nuevos estilos de vida, patrones culturales y de consumo que tienen en la gentrificación una de sus principales expresiones urbanas.

La segunda, cuyo autor más representativo es Smith $(1982,1986)$, propone una explicación de la gentrificación basada en la oferta, como parte de un proceso más general de reestructuración económica y espacial del modo de producción capitalista. Su tesis de la "brecha en la renta del suelo urbano" implica que la gentrificación se produce cuando la brecha entre la renta capitalizada por el uso actual y la renta potencial con un uso alternativo es suficientemente grande. Solo entonces la reinversión se vuelve económicamente rentable para una parcela determinada.

Uno de los elementos centrales del debate sobre la gentrificación es el rol del Estado en el desarrollo del proceso. Hacksworth y Smith (2001) reconocen tres oleadas de gentrificación diferentes. La primera, 1960-1970, originalmente captada por los trabajos pioneros de Glass, estaba caracterizada por procesos de gentrificación aislados, limitados, donde algunos gentrificadores pioneros daban sus primeros pasos. La segunda, 1970-1980, estuvo caracterizada por una extensión de la gentrificación a otras grandes ciudades, donde el Estado se limita a una política de laissez faire, sin intervenir mayormente en la dinámica del proceso. Y finalmente, desde 1990 hasta la actualidad, el proceso ha estado signado por una gran intervención del Estado en asociación con el capital privado, que registra una mayor presencia de capital concentrado corporativo, en detrimento de pequeños y medianos capitales. Wacquant (2008) señala que, en esta tercera oleada, el Estado pasa de ocupar un rol de proveedor de bienes y servicios para la población de bajos ingresos, a constituirse en proveedor de servicios para negocios, e instalaciones y servicios para sectores medios y altos urbanos.

El concepto de gentrificación fue acuñado en un tiempo y lugar específicos, lo que requiere para su utilización en otras latitudes una adaptación crítica. En esta línea, Herzer, Di Virgilio y Rodríguez (2011) señalan que, a diferencia de los procesos originales, en las ciudades de Latinoamérica no se registró el despoblamiento y decadencia económica de las áreas centrales que caracterizó a los primeros. Al contrario, las áreas centrales mantuvieron su vitalidad como centros administrativos y económicos, incluso fueron el centro de desarrollo de una fuerte cultura popular, pese a una relativa pérdida de población. El momento de desarrollo de las transformaciones en las áreas centrales también fue diferente: las intervenciones en áreas centrales se dieron en Norteamérica y Europa desde 1930, con especial énfasis durante la posguerra, mientras que dichos procesos comienzan en Latinoamérica a partir de la década de 1970 , precedidos por una revitalización y recuperación del valor patrimonial de los centros históricos coloniales. Otra diferencia central es que el Estado desempeñó un rol preponderante en la renovación urbana de las ciudades de América Latina, con una menor participación del sector privado que en otras latitudes.

En un sentido similar, Inzulza-Contardo (2011) presenta al menos tres características específicas de la gentrificación en ciudades latinoamericanas. Por una parte, el cambio de población que se verifica se expresa en el reemplazo de familias de pocos 
recursos por jóvenes profesionales inquilinos de ingresos medios -light-blue collar, según el autor-, a diferencia del cambio más drástico que se daría en procesos de gentrificación en países centrales. Esta evidencia es coincidente con la investigación realizada por Herzer, Di Virgilio, Guevara, Ramos y Vitale (2011), que describen "procesos de movilidad de tramos cortos", donde sectores vulnerables son desplazados por sectores medios-bajos, solo relativamente más consolidados en términos de inserción ocupacional. Por otra parte, en los barrios en proceso de gentrificación se verifican, por un lado, cambios físicos asociados a la intensificación de uso del suelo, que derivan en una mayor densificación a través de la sustitución de viviendas unifamiliares por edificios de densidad media y alta; y finalmente, cambios de uso que implican el reemplazo de inmuebles con fines residenciales por comercios, elemento que también es señalado por Herzer, Di Virgilio y Rodríguez (2011).

Esta vocación por interrelacionar las transformaciones del espacio urbano y los diferentes sectores sociales, emparenta de manera definitiva el abordaje de la gentrificación con el de la segregación. No obstante, la segregación, al enfatizar las dinámicas sociales y económicas vis-à-vis el espacio urbano, es en algún sentido la contracara de los estudios sobre gentrificación y difusión urbana, en el sentido de que estos últimos parten de una serie de transformaciones territoriales, a las que imputan una determinada dinámica socioeconómica, mientras que los primeros tienen como punto de partida la configuración y la dinámica de localización de los grupos sociales y su vinculación con la configuración del espacio urbano.

\section{Procesos de difusión urbana}

Existe una segunda sublínea de investigación, que complementa la anterior y enfatiza las transformaciones que se producen por la expansión acelerada de las ciudades, destacando el tipo de urbanización que se genera, su morfología, vinculación funcional, etcétera. Esta sublínea acuñó muchos términos diversos: ciudades difusas, ciudades de borde, periurbanización, metápolis, rururbanización, etcétera (Rufi, 2003). Si bien existen diferencias entre todos estos fenómenos, vamos a encuadrarlos bajo el término de difusión urbana, porque creemos que sintetiza adecuadamente muchas de las características observadas en las nuevas formas de expansión urbana señaladas. Las especificidades de los diferentes términos tienen que ver con los contextos en que son acuñados, la morfología particular de estos fenómenos, la disciplina desde donde se los aborda y el acento en uno u otro de los elementos del proceso, pero remiten claramente a una matriz común en la cual se los puede agrupar.

La difusión urbana nace como una conceptualización estrechamente ligada a la experiencia de la ciudad de Los Ángeles, en particular, y de muchas ciudades estadounidenses en general. Al igual que otros modelos de urbanismo propuestos, la difusión urbana no es fácilmente exportable a otras latitudes por fuera de Norteamérica, pero tiene cierta validez como herramienta heurística para reflexionar sobre las transformaciones territoriales en curso.

En una sistematización de factores que impulsan el fenómeno de la difusión urbana, Ainstein (2008) incluyó, entre otros, los siguientes: transformaciones en la sensibilidad locacional generada por economías de escala y de aglomeración; profundización de la estratificación social y de la segregación territorial de la 
población; incremento de las tasas de motorización, de la congestión circulatoria; creciente estratificación de los valores inmobiliarios; incrementada participación económica del sector inmobiliario; profundización de la segmentación institucional; creciente desvinculación estatal de la gestión regional/urbana. La conclusión general de este autor es que la difusión urbana, en oposición a la metropolización tradicional, disminuye la eficiencia, la eficacia, la equidad y la sustentabilidad del sistema urbano en su conjunto (Ainstein, Kralich, Villadeamigo \& Guevara, 2012).

La mayor parte de los trabajos parecen coincidir en las consecuencias negativas de los procesos de difusión urbana con respecto a la sustentabilidad ambiental y a la equidad de las ciudades, lo que plantea interrogantes sobre la posibilidad de mantener este ritmo de expansión urbana de baja densidad. Se plantea la necesidad de revertir estas tendencias, pero no parece haber indicios claros sobre cómo contrarrestarlas sin caer en recetas fallidas, como la planificación urbana de corte racionalista.

En términos más generales, los procesos de difusión urbana se relacionan con cambios más globales en el sistema económico, no solo con rupturas o continuidades en las tendencias de localización residencial. Ello ha ocurrido especialmente con el advenimiento de un modo de producción posfordista, que se caracteriza por la relocalización del aparato productivo, facilitado por las nuevas tecnologías de información y comunicación (Monclús, 1998).

El análisis de los fenómenos de difusión urbana en ciudades latinoamericanas contemporáneas requiere adaptaciones y críticas para su utilización. En estas ciudades, la suburbanización tuvo originalmente como protagonistas a los sectores de menos recursos, a diferencia de las ciudades norteamericanas, proceso que Torres (2001) denominó "suburbanización de los sectores populares" para el caso de Buenos Aires. Tales procesos explican una gran parte del crecimiento metropolitano de las ciudades latinoamericanas durante el siglo XX. Estas tendencias, marcadas por la extendida presencia de mecanismos informales de urbanización (Clichevsky, 2003), se mantienen hasta la actualidad, cada vez más alejadas de los centros urbanos tradicionales. En las últimas décadas, a los procesos de suburbanización popular se superponen otros protagonizados por sectores de mayores ingresos (el caso prototípico es el de las nuevas urbanizaciones cerradas), lo que promueve una fragmentación de la estructura urbana y segregación social a menor escala en las ciudades latinoamericanas.

Para cerrar esta sección, quisiéramos reflexionar de manera conjunta tanto sobre las limitaciones de los enfoques centrados en los procesos de gentrificación, como sobre los déficit de aquellos que apuntan a la difusión urbana. Se puede afirmar que ambas líneas de indagación comparten un sesgo que podríamos llamar morfológico, especialmente claro en el caso de la difusión urbana, menor en el caso de la gentrificación. Tal sesgo es que se interesan básicamente por identificar nuevas morfologías o formas urbanas, y a partir de esa identificación desprenden un conjunto de procesos sociales y económicos que le darían sustento. Ambos fenómenos son asociados con el proceso de globalización en curso, casi como las dos caras de una misma moneda: reconfiguración de áreas centrales y crecimiento y expansión en la periferia. No obstante, estos procesos tienen distintos protagonistas y lógicas diversas en las diferentes latitudes. Por ejemplo, los sectores de mayores ingresos 
son actores principales en la periurbanización, tanto como lo son los sectores de más bajos ingresos en las ciudades periféricas de Latinoamérica.

En definitiva, como veremos más adelante, parece más prudente inscribir todos estos procesos como reestructuraciones territoriales que se explican, de un lado, por los movimientos de inversión de capitales y sus respectivos ciclos de acumulación; del otro, por los procesos de producción social del hábitat vinculados a los sectores populares. Esto no implica quedarse en un nivel elevado de abstracción, sino al contrario, mantener un cierto orden de prelación entre los fenómenos abordados. De lo contrario, continuamente se están identificando nuevas formas urbanas y queriendo deducir de ellas procesos sociales, económicos, culturales y políticos. Por ello, es necesario inscribir todos estos procesos en un marco más general, donde los actores sociales se ponen en juego con sus lógicas, intereses y disposiciones que los constituyen como tales. En este marco, desarrollos de "alcance medio" como la gentrificación y la difusión urbana sirven como herramientas heurísticas para pensar diferentes contextos y sus especificidades locales.

\section{Reestructuración territorial}

En este trabajo, se propone enmarcar las transformaciones reseñadas más arriba bajo la perspectiva del materialismo histórico-geográfico, siguiendo fundamentalmente los trabajos de Harvey sobre la reestructuración territorial del capitalismo. Harvey (2003) cuestiona el presunto carácter novedoso de la etapa de globalización en curso, considerando que no se han modificado radicalmente el modo de producción y las relaciones sociales correspondientes. Antes bien, identifica esta etapa con un proyecto geopolítico específico, hegemonizado por los Estados Unidos y el capital financiero internacional. Lo describe de la siguiente manera:

La verdadera tendencia cualitativa es la reafirmación de los valores capitalistas del siglo XIX unidos a la tendencia del siglo XXI a arrastrar a todos (y a todo lo que se pueda intercambiar) a la órbita del capital, al tiempo que se hace que grandes segmentos de la población mundial sean permanentemente superfluos en relación con la dinámica básica de acumulación de capital. (Harvey, 2003, p. 87)

Empero, reconoce que la magnitud y velocidad de los cambios cuantitativos terminan por generar transformaciones cualitativas relevantes. La profunda reorganización espacial del sistema capitalista en las últimas décadas tuvo como una de sus consecuencias el debilitamiento de las unidades geográficas que acompañaron la consolidación del sistema, los Estados nacionales. Sin embargo, de acuerdo con su teoría de los desarrollos geográficos desiguales, la reconfiguración de las escalas geográficas de forma permanente es una de las características de la producción del espacio inherente al sistema capitalista, tanto como la producción de desigualdades geográficas.

Desde esta perspectiva, Harvey (2001) explica la reestructuración de las ciudades y regiones urbanas contemporáneas como una salida estructural de las recurrentes crisis capitalistas de sobreacumulación. Si bien las manifestaciones que identifica son similares a las mencionadas por los autores reseńados más arriba, sostiene que la determinación fundamental de los procesos de reestructuración está dada por 
la necesidad de superar los obstáculos que las recurrentes crisis generan para la acumulación capitalista. Las crisis regulan y ordenan el desarrollo, con un coste social y económico muy alto, pero también sientan las bases para continuar con el proceso de acumulación. Permiten absorber los excedentes de capital y trabajo generados por la sobreacumulación endémica. La constante expansión y reestructuración geográfica se inserta, así, en una perspectiva estructural del sistema capitalista y sus contradicciones inherentes. El paisaje producido por el capitalismo no está exento de ellas, y así como posibilita el despliegue de la acumulación, rápidamente se convierte en obstáculo y entra en contradicción con el desarrollo de las fuerzas productivas. De ahí que requiera su reestructuración permanente e incluso a una velocidad cada vez más acelerada. La noción de destrucción creativa que emplea Harvey (2005) permite aprehender esta lógica de producción del espacio bajo el sistema capitalista. Es la lógica que orienta los procesos que el autor denomina "ajustes espacio-temporales": la idea básica es que la sobreacumulación cíclica del capitalismo supone excedentes de trabajo y capital que pueden ser absorbidos de diferentes maneras: a) el desplazamiento temporal a través de inversiones de capital y trabajo en proyectos de largo plazo o gastos sociales, como pueden ser grandes infraestructuras o equipamientos; b) desplazamientos espaciales a través de la apertura de nuevos mercados, nuevas capacidades productivas y nuevas posibilidades de recursos y de trabajo en otros lugares; c) alguna combinación de los dos anteriores, ambas con impactos muy fuertes en términos de la estructura territorial.

De acuerdo con este autor, la "acumulación por desposesión" (Harvey, 2005) es uno de los más recientes mecanismos de ajuste espacio-temporal en la reestructuración territorial del capitalismo en curso. Esta nueva forma de imperialismo implica una alianza entre los poderes estatales, con su monopolio de la violencia y sus definiciones de legalidad y los aspectos depredadores del sistema financiero, en contra de la voluntad popular de las mayorías. Esta forma extrema y cada vez más violenta de dominación está expresando la crisis del modelo de acumulación instaurado en la década de 1970. La acumulación por desposesión implica en alguna medida el fracaso del proyecto de la globalización hegemonizada por los Estado Unidos. Esta se basa en la libre circulación de capitales y la apertura comercial indiscriminada, que fue promovida por organismos como el Fondo Monetario Internacional (FMI) y el Consenso de Washington. La crisis de 2008 mostró con claridad el agotamiento de este modelo, que según Harvey ya venía acusando un deterioro creciente, pese a que el dominio financiero de los Estados Unidos había permitido ocultarlo, generando desequilibrios macroeconómicos mayúsculos en su economía. En la actualidad, la crisis persiste y trasladó su centro a los países de Europa, jaqueando el proceso de integración regional del continente. La contracara de este deterioro es la creciente militarización, como ultima ratio de la debilitada hegemonía norteamericana, cuyo objetivo es el control de las reservas de hidrocarburos mundiales y otros recursos naturales estratégicos. La resolución de la presente crisis es un interrogante, pero el autor afirma que, en este escenario, los mecanismos de acumulación por desposesión cobran un mayor peso relativo, con todas las consecuencias sociales y políticas negativas que traen aparejadas. Estos mecanismos están completamente vigentes en las sociedades latinoamericanas y explican en gran medida el auge de los conflictos 
vinculados al acceso al suelo, tanto rural (por las trasnformaciones en el agro) como urbano (por las transformaciones reseńadas en este trabajo).

En su trabajo más reciente, de 2012, Harvey va un paso más allá y establece la vinculación estructural entre las crisis de sobreacumulación y el sector inmobiliario. Este sector mantiene una relación estrecha con el capital financiero, del cual depende para su mera subsistencia, dado el elevado tiempo de circulación de capital y de realización de las mercancías en este sector. No obstante, la naturaleza del capital financiero, en especial en esta etapa de su avanzada mundialización (Pradilla Cobos, 2009), hace que a largo plazo primen los comportamientos especulativos que refuerzan la tendencia a la sobreacumulación en el sector. De ahí que Harvey (2012) identifique en el origen de muchas de las crisis de sobreacumulación del sistema capitalista, una crisis gestada inicialmente en el sector inmobiliario y que es trasladada al conjunto del sistema a través del capital financiero. Este mecanismo vale tanto para crisis tan paradigmáticas como la de 1930, como para la más reciente de 2008, nacida al calor del colapso de las denominadas hipotecas subprime. Kozulj (2003), en un abordaje complementario, señala justamente que una de las claves del proceso de expansión económica del período de la segunda posguerra tuvo que ver con el desarrollo de un paquete tecnológico vinculado a la producción de las ciudades, es decir, al proceso de urbanización entendido de forma global.

Esta perspectiva teórica más general de la reestructuración territorial del sistema capitalista permite enmarcar los llamados procesos de globalización en uno más amplio y de larga data, vinculado a la dinámica expansiva inherente al sistema capitalista. A su vez, pone de manifiesto la importancia de la reestructuración geográfica del aparato productivo, que trae aparejada la emergencia de nuevos centros de producción, tanto dentro de las economías centrales (Silicon Valley), como externos (el ascenso del eje productivo del Sudeste Asiático). La contracara de esta reestructuración productiva es una nueva especialización de las economías urbanas de las ciudades de los países centrales que, ante la caída de empleo industrial, se concentran en los servicios de la producción, como enfatiza el abordaje de las ciudades globales. Claro que este nuevo sector dinámico no compensa la pérdida de empleo en el sector industrial, lo que produce la segmentación de los mercados laborales, identificada por diversos autores, y la expansión del desempleo, el subempleo y la precarización laboral que puede identificarse en muchos países centrales en la actualidad. La noción de dualización de la economía es una sobresimplificación, no adecuada para abarcar la complejidad de este proceso de permanente reestructuración que expresa la dialéctica de lo global y lo local, donde ninguna de las escalas ejerce una determinación completa sobre la otra Esto permite comprender cómo en las ciudades latinoamericanas contemporáneas puede darse de manera simultánea la profundización de problemáticas estructurales previas, como la precaria inserción en el aparato productivo de una parte importante de la población -lo que la escuela de la marginalidad denominaba polo marginal (Quijano, 1998) - y la industrialización parcial y precaria de otras áreas a partir de la modalidad de la maquila o el ensamblaje.

En este marco general, gentrificación y difusión urbana aparecen como dos expresiones territoriales del régimen de acumulación capitalista vigente en su fase de reestructuración, estrategias para garantizar dicha acumulación a través 
de mecanismos de ajuste espacio-temporal. En tanto procesos sociales y urbanos concretos, no pueden ser generalizados a priori, sino que su desarrollo o no desarrollo depende estrechamente de la estructura urbana preexistente, de los procesos de urbanización y producción del hábitat, del rol desempeñado por el Estado, etcétera. En este sentido, se ubican en un nivel de abstracción menor a la lógica de estructuración capitalista del territorio señalada por Harvey, que operaría como un determinante teórico fundamental (Jaramillo, 2009). De ahí que en las ciudades latinoamericanas contemporáneas, por ejemplo, las áreas centrales mantuvieran mayor vitalidad como centros administrativos, económicos y en algunos casos residenciales, a diferencia de la suburbanización norteamericana. De la misma forma, el modelo de suburbio blanco de clase media de EE.UU. poco tiene que ver con la suburbanización y periurbanización de las elites en nuestra región, donde muchas veces conviven en un área acotada urbanizaciones cerradas residenciales, con barrios populares de urbanización informal. Las conceptualizaciones que intentan capturar estos procesos sociales y urbanos podrían ser consideradas teorías de alcance intermedio, siguiendo la conocida clasificación de Merton (1964): su valor depende de que se especifique adecuadamente el tipo de formación social y estructura urbana en el que tienen vigencia. Asimismo, son conceptualizaciones valiosas como herramientas heurísticas que nos permiten contrastar los procesos concretos con procesos "típicos" desarrollados en otras latitudes, pero no son tendencias generalizables.

En este sentido, es importante centrarse en la noción de "solución espacial" que formula Harvey (2001), como una de las formas de resolución (temporal) de las recurrentes crisis de sobreacumulación de capital. Esta solución espacial es el resultado de la contradicción entre el proceso económico -las fuerzas productivas- y las estructuras físicas generadas en momentos previos. La inmovilidad de una porción del capital y de la fuerza de trabajo en determinadas infraestructuras fijas permite asegurar la movilidad del capital y de la fuerza de trabajo en el resto del circuito de acumulación. No obstante, el desarrollo de las fuerzas productivas entra rápidamente en contradicción con ese paisaje construido y requiere su permanente reformulación, con el resultado de que ninguna configuración regional o urbana persiste demasiado en el tiempo. De aquí se deriva la lógica permanente de transformación territorial que se evidencia en las ciudades latinoamericanas contemporáneas, que no tiene tanto que ver con las necesidades de habitación o empleo de la población, sino que muchas veces sigue la misma lógica de valorización del capital. En este sentido, De Mattos (2007) señala la convergencia entre capital financiero y capital inmobiliario a partir de instituciones financieras especializadas como un elemento central para comprender la transformación de las ciudades latinoamericanas contemporáneas. Es por eso que Pírez (2006) destaca esta convergencia como la evidencia de una verdadera privatización del desarrollo urbano, que refiere a la primacía de procesos de producción del hábitat orientados por la lógica de la ganancia. 


\section{Conclusiones}

En este artículo se propuso una sistematización de los diferentes abordajes teóricos sobre las transformaciones sociales, económicas y territoriales en las ciudades latinoamericanas contemporáneas. Para ello, se postuló la necesidad de comprender dichas transformaciones en un marco más general que el de esos enfoques particulares. Se optó para ello por una aproximación centrada en la reestructuración territorial del sistema capitalista, que ha entrado en una nueva etapa del proceso de mundialización. Esta línea implica poner de relieve lo territorial como una dimensión fundamental de la dinámica del sistema capitalista, dimensión que está atravesada por una contradicción -que le es inherente- entre el espacio construido y las siempre renovadas necesidades espaciales del proceso de acumulación. La resolución de esta contradicción se produce, en gran medida, a través del despliegue de diversas formas de destrucción creativa del espacio, que no son otra cosa que las transformaciones territoriales identificadas en los centros urbanos latinoamericanos contemporáneos, las cuales cumplen un rol fundamental en términos de absorción de excedentes de capital y trabajo y tienden así a asegurar la reproducción del proceso de acumulación. Tales cambios deben ser incorporados en los análisis históricos y geográficos específicos en el marco de teorías de alcance medio dentro del esquema conceptual general propuesto.

Esta reestructuración territorial del sistema capitalista implica, entre otros elementos, la relocalización del aparato productivo, donde se destaca la emergencia del polo del sudeste asiático, pero también la relocalización parcial de industrias de maquila y ensamblaje en ciudades grandes e intermedias de Latinoamérica. Esta globalización sesgada de los territorios latinoamericanos profundiza y complejiza la estructura social a través del mercado laboral como principal vector, extendiendo los fenómenos del desempleo, subempleo, precarización, etcétera. En este marco, las transformaciones reseñadas son comprendidas como parte de los mecanismos de ajuste espacio-temporal que genera el sistema como "salida" a las recurrentes crisis de sobreacumulación y como forma de reanudar el ciclo de acumulación de capital. Esto determina la emergencia de los procesos de segregación, gentrificación y difusión urbana analizados a lo largo del artículo. Tal perspectiva permite integrar los procesos socioespaciales específicos de las ciudades latinoamericanas contemporáneas en una lógica de transformación y producción del territorio más general. De la misma manera, da pie para comprender las transformaciones en las formas de gestión urbana por parte del Estado, que en términos generales denotan una crisis de la planificación racional y su transición a la planificación estratégica como herramienta central del urbanismo globalizador que privatiza el desarrollo urbano. 


\section{Referencias bibliográficas}

Ainstein, L. (2008, septiembre). A systemic approach to urban sprawl: distinguishing traits, underlying factors, emerging consequences and demanded public policies [Una aproximación sistémica a la difusión urbana: trazos distintivos, factores subyacentes, consecuencias emergentes y políticas públicas demandadas]. Ponencia presentada en 44th. Congress of the International Society of City and Regional Planners (IsoCaRP). Dalian, China.

Ainstein, L., Kralich, S., Villadeamigo, J. \& Guevara, T. (2012). Una modelización comparada relativa a Grandes Aglomerados Compactos y Grandes Aglomeraciones Difusas. En L. Ainstein (Coord.), Estructuración urbana, institucionalidad y sustentabilidad de ciudades metropolitanas y regiones difusas. Miradas comparadas de Buenos Aires, Londres, Los Ángeles, París, Tokio, Toronto (pp. 33-75). Buenos Aires: Editorial Universidad de Buenos Aires (Eudeba).

Borja, J. \& Castells, M. (con la colaboración de M. Belil y Ch. Benner) (1997). Local y global: la gestión de las ciudades en la era de la información. Madrid: United Nations for Human Settlements (Habitat); Taurus.

Castells, M. (1995). La ciudad informacional. Tecnologias de la información, reestructuración económica y el proceso urbano regional. Madrid: Alianza Editorial.

Castells, M. \& Mollenkopf, J. (Eds.). (1992). Dual city: Restructuring New York. Nueva York: Russell Sage Foundation.

Clichevsky, N. (2003). Pobreza y acceso al suelo urbano. Algunas interrogantes sobre las políticas de regularización en América Latina. Serie Medio ambiente y desarrollo n. ${ }^{\circ} 76$. Santiago: Comisión Económica para América Latina y el Caribe (CEPAL). Disponible en http:// repositorio.cepal.org/bitstream/handle/11362/5780/S0311860_es.pdf?sequence=1

De Mattos, C. (2002). Redes, nodos y ciudades: transformación de la metrópoli latinoamericana. Ponencia presentada en VII Seminario Internacional de la Red Iberoamericana de Investigadores sobre Globalización y Territorio. Camagüey, Cuba, 27-29 noviembre 2002. Disponible en http://es.scribd.com/doc/84525919/Carlos-de-Mattos-RedesNodos-y-Ciudades-Transf-de-La-Metropoli-a\#scribd

De Mattos, C. (2007, noviembre-diciembre). Globalización, negocios inmobiliarios y transformación urbana. Revista Nueva Sociedad, (212), 82-96. Disponible en http:// www.nuso.org/revista.php? $\mathrm{n}=212$

Engels, F. ([1848] 1965). La situación de la clase obrera en Inglaterra. Buenos Aires: Futuro.

Friedmann, J. (1972). A generalized theory of polarized development. En N. Hansen (Dir.), Growth centers in regional economic development. Nueva York: Free Press.

Groisman, F. \& Suárez, A. (2006, octubre). Segregación residencial en la Ciudad de Buenos Aires. Población de Buenos Aires. Revista semestral de datos y estudios sociodemográficos, 3(4), 27-37. Disponible en http://www.redalyc.org/articulo.oa?id=74030403

Hacksworth, J. \& Smith, N. (2001). The changing state of gentrification. Tijdschrift voor economische en sociale geografie, 92(4), 464-477. doi: 10.1111/1467-9663.00172

Hamnett, C. (1991). The blind men and the elephant: The explanation of gentrification. Transactions of the Institute of British Geographers, New Series, 16(2), 173-189. Disponible en http://homepages.neiu.edu/ -dgrammen/1991HAMNETT.pdf 
Harvey, D. (1998). La condición de la posmodernidad. Investigación sobre los origenes del cambio cultural. Buenos Aires: Amorrortu Ediciones.

Harvey, D. (2001). Espacios del capital. Hacia una geografía crítica. Madrid: Akal.

Harvey, D. (2003). Espacios de esperanza. Madrid: Akal.

Harvey, D. (2005, enero). El "Nuevo" Imperialismo: Acumulación por desposesión, Socialist Register 2004 (pp. 99-129). Buenos Aires: Consejo Latinoamericano de Ciencias Sociales (CLACso). Disponible en http://biblioteca.clacso.edu.ar/gsdl/collect/clacso/ index/assoc/D8555.dir/harvey.pdf

Harvey, D. (2012). Rebel cities: From the right to the city to urban revolution. Londres: Verso.

Herzer, H., Di Virgilio, M. \& Rodríguez, C. (2011, agosto-septiembre). Gentrification in Buenos Aires City: A drip strategy. Ponencia presentada en RGS-IBG 2011 Conference: The Geographical Imagination. Londres, 31 agosto-2 septiembre 2011.

Herzer, H., Di Virgilio, M., Guevara, T., Imori, M., Ramos, J. \& Vitale, P. (2011, octubre). Unos llegan, otros se van: cambios y permanencias en el barrio de La Boca. Población de Buenos Aires. Revista semestral de datos y estudios sociodemográficos, 8(14), 7-28. Disponible en http://www.buenosaires.gob.ar/areas/hacienda/sis_estadistico/ poblacion_2011_014.pdf

Ibarra Cofré, R. (2007). Segregación socio-espacial en ciudades turísticas. El caso de Canela (RS), Brasil. Estudios y Perspectivas en Turismo, 16(2), 195-215. Disponible en http:// www.redalyc.org/articulo.oa?id=180713889004

Inzulza-Contardo, J. (2011). "Latino-gentrification"? Focusing on physical and socioeconomic patterns of change in Latin American inner cities. Urban Studies, 14. doi:10.1177/0042098011423425

Jaramillo, S. (2009). Hacia una teoría de la renta del suelo urbano. Bogotá: Universidad de Los Andes.

Kaztman, R. (1999). Marco conceptual sobre activos, vulnerabilidad y estructura de oportunidades. Montevideo: Comisión Económica para América Latina y el Caribe (CEPAL). Disponible en http://www.eclac.org/publicaciones/xml/6/10816/LC-R176.pdf.

Kaztman, R. (2001). La dimensión espacial en las politicas de superación de la pobreza urbana. Serie Medio Ambiente y Desarrollo 59. Santiago: Comisión Económica para América Latina y el Caribe (CEPAL). Disponible en http://repositorio.cepal.org/bitstream/ handle/11362/5761/S0210799_es.pdf?sequence $=1$

Kozulj, R. (2003, enero). Urbanización, cambio tecnológico y sobrecapacidad estructural: de los años dorados a la globalización. Comercio Exterior, 53(1), 24-41. Disponible en http://revistas.bancomext.gob.mx/rce/magazines/14/3/RCE.pdf

Lanzetta, M. (2011). Christian Topalov: la emergencia de la línea de pobreza y la cartografía social. Apuntes de Investigación del CECyP, (16/17), 245-257. Disponible en http:// www.apuntescecyp.com.ar/index.php/apuntes/article/view/310

Ley, D. (1986). Alternative explanations for inner-city gentrification: A Canadian assessment. Annals of the Association of American Geographers, 76(4), 521-535. doi: 10.1111/ j.1467-8306.1986.tb00134.x

Marx, K. \& Engels, F. (1848). Manifiesto del Partido Comunista. [Edición electrónica. Buenos Aires, 2004]. Disponible en http://archive.is/5L0dY

Merton, R.K. (1964). Teoría y estructura sociales. México, DF: Fondo de Cultura Económica. 
Monclús, F. (1998). Introducción: Suburbanización y nuevas periferias. Perspectivas geográficourbanísticas. En F. Monclús (Ed.), La ciudad dispersa (pp. 5-15). Barcelona: Centro de Cultura Contemporània de Barcelona. Disponible en http://archivouel.tripod.com/ dispersa.pdf

Pírez, P. (2006). La privatización de la expansión metropolitana en Buenos Aires. Economía, Sociedad y Territorio, 6(21), 31-54. Disponible en http://www.redalyc.org/articulo. oa?id $=11162103$

Pradilla Cobos, E. (2009). Los territorios del neoliberalismo. Compilación de ensayos. México, DF: Miguel Ángel Porrúa.

Quijano, A. (1998). La economía popular y sus caminos en América Latina. Lima: Mosca Azul.

Rodríguez Merkel, G. (2011). Desigualdades socioeconómicas y segregación residencial en la Argentina, 1991-2001. Niveles, tendencias y aportes teórico-metodológicos para su estudio. Disertación Tesis Doctoral. Universidad de Buenos Aires.

Rodríguez Vignoli, J. (2001). Segregación residencial socioeconómica: ¿qué es?, ¿cómo se mide?, ¿qué está pasando?, ¿importa? Serie Población y Desarrollo, 16. Santiago: Comisión Económica para América Latina y el Caribe (CEPAL). Disponible en http://repositorio. cepal.org/bitstream/handle/11362/7149/S017595_es.pdf?sequence $=1$

Rufi, J.V. (2003). ¿Nuevas palabras, nuevas ciudades? Revista de Geografía (Universitat de Barcelona), (2), 79-103. Disponible en http://www.raco.cat/index.php/ RevistaGeografia/article/view/46133

Sabatini, F. (1999). Tendencias de la segregación residencial urbana en Latinoamérica: Reflexiones a partir del caso de Santiago de Chile. Documentos del Instituto de Estudios Urbanos, Serie Azul, 29. P. Universidad Católica de Chile.

Sabatini, F., Brain, I. (2008). La segregación, los guetos y la integración social urbana: mitos y claves. EURE, 34(103), 5-26. http://dx.doi.org/10.4067/S0250-71612008000300001

Sabatini, F., Cáceres, G. \& Cerda, J. (2001). Segregación residencial en las principales ciudades chilenas: tendencias de las últimas tres décadas y posibles cursos de acción. EURE, 27(82), 21-42. http://dx.doi.org/10.4067/S0250-71612001008200002

Sassen, S. (1997). Las ciudades en la economía global. Ponencia presentada en Simposio de Ciudades, Barcelona, 13-15 de marzo de 1997. [Thousand Oaks, Ca: Pine Forges Press, 2000].

Sassen, S. (2007). Una sociología de la globalización. Buenos Aires: Editorial Katz.

Sassen, S. (2010). Territorio, autoridad y derechos. De los ensamblajes medievales a los ensamblajes globales. Buenos Aires: Editorial Katz.

Slater, T. (2011). Gentrification of the city. En G. Bridge \& S. Watson. The New Blackwell Companion to the City (pp. 571-585). Oxford, un: Wiley-Blackwell. doi: $10.1002 / 9781444395105 . \operatorname{ch} 50$

Smith, N. (1982). Gentrification and uneven development. Economic Geography, 58(2), 139-155.

Smith, N. (1986). Gentrification and the rent gap. Annals of the Association of American Geographers, 77(3), 462-465. doi: 10.1111/j.1467-8306.1987.tb00171.x

Soja, E. (2008). Postmetrópolis. Estudios Críticos sobre las ciudades y las regiones. Madrid: Traficantes de Sueños.

Spengler, O. (1918). La decadencia de Occidente. [Edición electrónica. Buenos Aires, 2006]. Disponible en http://disenso.info/wp-content/uploads/2013/06/La-Decadencia-deOccidente-O.-Spengler.pdf 
Suárez, A. (2005, agosto). Segregación residencial y pobreza. Consecuencias del aislamiento social de residentes en asentamientos precarios. Ponencia presentada en el XXV Congreso de la Asociación Latinoamericana de Sociología, Porto Alegre, Brasil.

Torres, H. (2001). Cambios socioterritoriales recientes en Buenos Aires durante la década de 1990. EURE, 2(8), 33-56. http://dx.doi.org/10.4067/S0250-71612001008000003

Wacquant, L. (2007). Los condenados de la ciudad. Gueto, periferias y Estado. Buenos Aires: Siglo xxi Editores.

Wacquant, L. (2008). Relocating gentrification: The working class, science and the state in recent urban research. International Journal of Urban and Regional Research, 32(1), 198-205. doi: 10.1111/j.1468-2427.2008.00774.x

Wacquant, L. (2010). Las dos caras de un gueto. Ensayos sobre marginalización y penalización. Buenos Aires: Siglo xxi Editores.

Ward, P. (2001). Squaring the circle: Whither or wither segregation in Latin American cities? Ponencia presentada en el International Seminar on Segregation in the City. Cambridge, MA, Lincoln Institute for Land Policy, 26-28 julio 2001. 